\title{
Complete genome sequence of Chitinophaga pinensis type strain (UQM 2034')
}

\author{
Tijana Glavina Del Rio ${ }^{1}$, Birte Abt $^{2}$, Stefan Spring ${ }^{2}$, Alla Lapidus ${ }^{1}$, Matt Nolan ${ }^{1}$, Hope Tice ${ }^{1}$, \\ Alex Copeland ${ }^{1}$, Jan-Fang Cheng ${ }^{1}$, Feng Chen ${ }^{1}$, David Bruce ${ }^{1,3}$, Lynne Goodwin ${ }^{1,3}$, Sam \\ Pitluck ${ }^{1}$, Natalia Ivanova ${ }^{1}$, Konstantinos Mavromatis ${ }^{1}$, Natalia Mikhailova ${ }^{1}$, Amrita Pati ${ }^{1}$, \\ Amy Chen ${ }^{4}$, Krishna Palaniappan ${ }^{4}$, Miriam Land ${ }^{1,5}$, Loren Hauser ${ }^{1,5}$, Yun-Juan Chang ${ }^{1,5}$, \\ Cynthia D. Jeffries ${ }^{1,5}$, Patrick Chain ${ }^{2,3}$, Elizabeth Saunders ${ }^{3}$, John C. Detter ${ }^{1,3}$, Thomas \\ Brettin $^{1,3}$, Manfred Rohde ${ }^{7}$, Markus Göker ${ }^{2}$, Jim Bristow ${ }^{1}$, Jonathan A. Eisen ${ }^{1,8}$, Victor \\ Markowitz ${ }^{4}$, Philip Hugenholtz ${ }^{1}$, Nikos C. Kyrpides ${ }^{1}$, Hans-Peter Klenk ${ }^{2 *}$, and Susan Lucas ${ }^{1}$ \\ ${ }^{1}$ DOE Joint Genome Institute, Walnut Creek, California, USA \\ ${ }^{2}$ DSMZ - German Collection of Microorganisms and Cell Cultures GmbH, Braunschweig, \\ Germany \\ ${ }^{3}$ Los Alamos National Laboratory, Bioscience Division, Los Alamos, New Mexico, USA \\ ${ }^{4}$ Biological Data Management and Technology Center, Lawrence Berkeley National \\ Laboratory, Berkeley, California, USA \\ ${ }^{5}$ Oak Ridge National Laboratory, Oak Ridge, Tennessee, USA \\ ${ }^{6}$ Lawrence Livermore National Laboratory, Livermore, California, USA \\ ${ }^{7}$ HZI - Helmholtz Centre for Infection Research, Braunschweig, Germany \\ ${ }^{8}$ University of California Davis Genome Center, Davis, California, USA
}

*Corresponding author: Hans-Peter Klenk

Keywords: filamentous, gliding, myxospores, aerobic, mesophile, Gram-negative, biomass degrader, chitinolytic, 'Chitinophagaceae', GEBA

Chitinophaga pinensis Sangkhobol and Skerman 1981 is the type strain of the species which is the type species of the rapidly growing genus Chitinophaga in the sphingobacterial family 'Chitinophagaceae'. Members of the genus Chitinophaga vary in shape between filaments and spherical bodies without the production of a fruiting body, produce myxospores, and are of special interest for their ability to degrade chitin. Here we describe the features of this organism, together with the complete genome sequence, and annotation. This is the first complete genome sequence of a member of the family 'Chitinophagaceae', and the 9,127,347 bp long single replicon genome with its 7,397 protein-coding and 95 RNA genes is part of the Genomic Encyclopedia of Bacteria and Archaea project.

\section{Introduction}

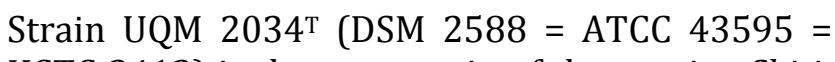
KCTC 3412) is the type strain of the species Chitinophaga pinensis and was first described in 1981 by Sangkhobol and Skerman [1]. In 1981, strain UQM 2034T was described as a long, filamentous, gliding microorganism isolated from an infusion of litter from the base of a pine tree in Alderley, Brisbane, Australia [1]. In 1999, the phylogenetic position of $C$. pinensis was determined. The comparison of $16 \mathrm{~S}$ rRNA sequences revealed Flexibac- ter filimoris as the most closely related bacterium [2].

In 2006 Kämpfer et al. reclassified F. sancti, F. filiformis, F. japonensis and Cytophaga arvensicola to the monospecific genus Chitinophaga and proposed C. skermanii sp. nov. [3]. In recent years the number of newly described species belonging to the genus Chitinophaga increased. Two additional new Chitinophaga species were described in 2007, C. ginsengisegetis sp. nov. and C. ginsengisoli sp. 
nov. isolated from soil of a ginseng field in South Korea [4]. In the same year Kim and Jung described the new species $C$. terrae sp. nov [5]. In 2009, three additional Chitinophaga species were described: $C$. niabensis sp. nov. [6], C. niastensis sp. nov [6], and $C$. rupis sp. nov [7]. Here we present a summary classification and a set of features for $C$. pinensis UQM 2034T , together with the description of the complete genomic sequencing and annotation.

\section{Classification and features}

The most similar 16S rRNA gene sequences from cultivated strains that are stored in GenBank originate from isolates belonging to different species of the genus Chitinophaga: C. sancti, C. filiformis and $C$. ginsengisoli with $96-97 \%$ sequence similari- ty; all of them were isolated from soil samples. In metagenomic surveys of environmental samples only 16S rRNA genes with sequence similarity values below $92 \%$ to $C$. pinensis were detected, indicating that members of this species are not abundant in the so far genomically screened habitats (status July 2009).

Figure 1 shows the phylogenetic neighborhood of C. pinensis UQM $2034^{\mathrm{T}}$ in a $16 \mathrm{~S}$ rRNA based tree. The sequences of the six copies of the $16 \mathrm{~S}$ rRNA gene in the genome differ by up to five nucleotides, and differ by up to 21 nucleotides (1.4\%) from the previously published sequence generated from ACM 2034 (AF78775). Most likely this discrepancy is caused by sequencing errors in the publicly available $C$. pinensis $16 \mathrm{~S}$ sequence.

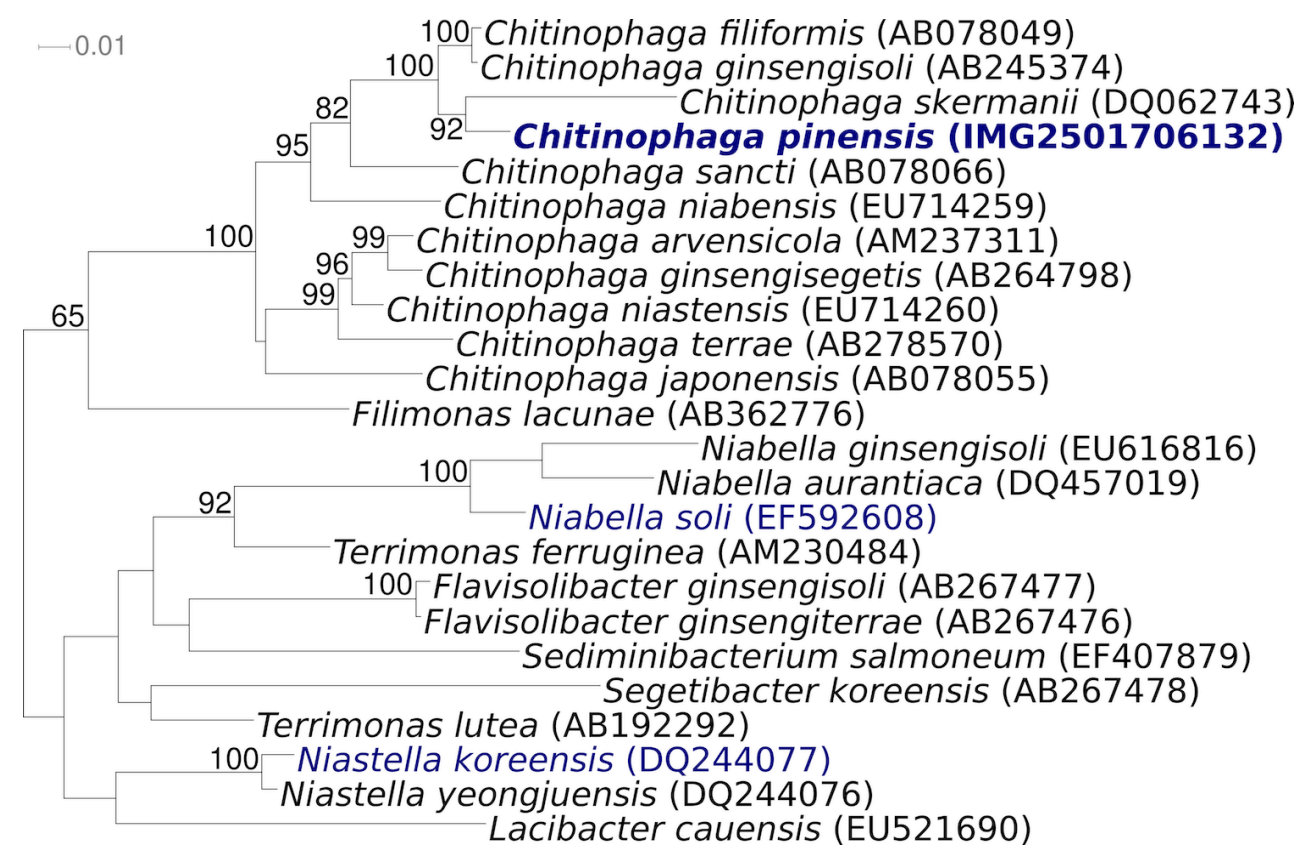

Figure 1. Phylogenetic tree highlighting the position of $C$. pinensis UQM $2034^{\top}$ relative to the other type strains within the genus and selected other type strains within the family 'Chitinophagaceae'. The tree was inferred from 1,410 aligned characters [8,9] of the 16S rRNA gene sequence under the maximum likelihood criterion [10] and rooted in accordance with the current taxonomy. The branches are scaled in terms of the expected number of substitutions per site. Numbers above branches are support values from 1,000 bootstrap replicates if larger than $60 \%$. Lineages with type strain genome sequencing projects registered in GOLD [11] are shown in blue, published genomes in bold.

Cells of $C$. pinensis stain Gram-negative and form long, filamentous, flexible rods with rounded ends (Table 1). They occur singly and measure 0.5-0.8 by $40 \mu \mathrm{m}$ in the mature gliding stage. Figure 2 shows cells of $C$. pinensis during cell division before separation. Upon aging, they transform into spherical bodies without the production of fruiting bodies. Myxospores with a diameter of 0.8-0.9 $\mu \mathrm{m}$ are formed. On charcoal yeast extract agar (CYEA) a yellowish pigment is produced. The temperature for growth ranges from $12-37^{\circ} \mathrm{C}$ with an optimum of $24^{\circ} \mathrm{C}$. The optimal $\mathrm{pH}$ for growth is 7 , but growth is possible in a wide $\mathrm{pH}$ range from 4 to $10 . \mathrm{NaCl}$ concentrations of $0-1.5 \%(\mathrm{w} / \mathrm{v})$ are tolerated [1]. 
Table 1. Classification and general features of C. pinensis UQM 2034 ${ }^{\top}$ according to the MIGS recommendations [12]

\begin{tabular}{|c|c|c|c|}
\hline MIGS ID & Property & Term & Evidence code \\
\hline & \multirow{8}{*}{ Current classification } & Domain Bacteria & TAS [13] \\
\hline & & Phylum Bacteroidetes & TAS [14] \\
\hline & & Class Sphingobacteria & TAS [14] \\
\hline & & Order Sphingobacteriales & TAS [14] \\
\hline & & Family 'Chitinophagaceae' & NAS \\
\hline & & Genus Chitinophaga & TAS [1] \\
\hline & & Species Chitinophaga pinensis & TAS [1] \\
\hline & & Type strain UQM 2034 & TAS [1] \\
\hline & Gram stain & negative & TAS [1] \\
\hline & Cell shape & filamentous & TAS [1] \\
\hline & Motility & gliding & TAS [1] \\
\hline & Sporulation & myxospores & TAS [1] \\
\hline & Temperature range & mesophile, $12-40^{\circ} \mathrm{C}$ & TAS [1] \\
\hline & Optimum temperature & $24^{\circ} \mathrm{C}$ & TAS [1] \\
\hline & Salinity & up to $1.5 \% \mathrm{NaCl}$ & TAS [1] \\
\hline \multirow[t]{3}{*}{ MIGS-22 } & Oxygen requirement & aerobic & TAS [1] \\
\hline & Carbon source & $\begin{array}{l}\text { acid production from glucose, } \\
\text { lactose and sucrose }\end{array}$ & TAS [1] \\
\hline & Energy source & chemoorganotrophic & TAS [1] \\
\hline MIGS-6 & Habitat & 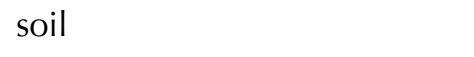 & TAS [1] \\
\hline MIGS-15 & Biotic relationship & free living & NAS \\
\hline \multirow[t]{3}{*}{ MIGS-14 } & Pathogenicity & non pathogenic & NAS \\
\hline & Biosafety level & 1 & TAS [15] \\
\hline & Isolation & pine litter & TAS [1] \\
\hline MIGS-4 & Geographic location & Alderley, Brisbane, Australia & TAS [1] \\
\hline MIGS-5 & Sample collection time & in 1981 or before & NAS \\
\hline $\begin{array}{l}\text { MIGS- } 4.1 \\
\text { MIGS- } 4.2\end{array}$ & $\begin{array}{l}\text { Latitude } \\
\text { Longitude }\end{array}$ & $\begin{array}{l}-27.424 \\
153\end{array}$ & TAS \\
\hline MIGS-4.3 & Depth & not reported & \\
\hline MIGS-4.4 & Altitude & not reported & \\
\hline
\end{tabular}

Evidence codes - IDA: Inferred from Direct Assay (first time in publication); TAS: Traceable Author Statement (i.e., a direct report exists in the literature); NAS: Non-traceable Author Statement (i.e., not directly observed for the living, isolated sample, but based on a generally accepted property for the species, or anecdotal evidence). These evidence codes are from of the Gene Ontology project [16]. If the evidence code is IDA, then the property was observed for a living isolate by one of the authors or an expert mentioned in the acknowledgements.

Strain UQM 2034T produces acid from glucose, lactose, and sucrose. Chitin, casein and gelatin are hydrolyzed, whereas according to Sangkhobol and Skerman (1981) cellulose, starch, alginate, and agar are not hydrolyzed [1]. Nitrate is not reduced to nitrite. C. pinensis UQM 2034T produces urease and is catalase and oxidase positive [1,3]. UQM
$2034^{\mathrm{T}}$ is susceptible to tetracycline, streptomycin, and chloramphenicol and resistant to neomycin, kanamycin, penicillin $\mathrm{G}$, and erythromycin [1]. $C$. pinensis UQM $2034^{\mathrm{T}}$ is able to lyse Staphylococcus aureus cells but not cells of Escherichia coli, Pseudomonas aeruginosa and Bacillus subtilis [1]. 


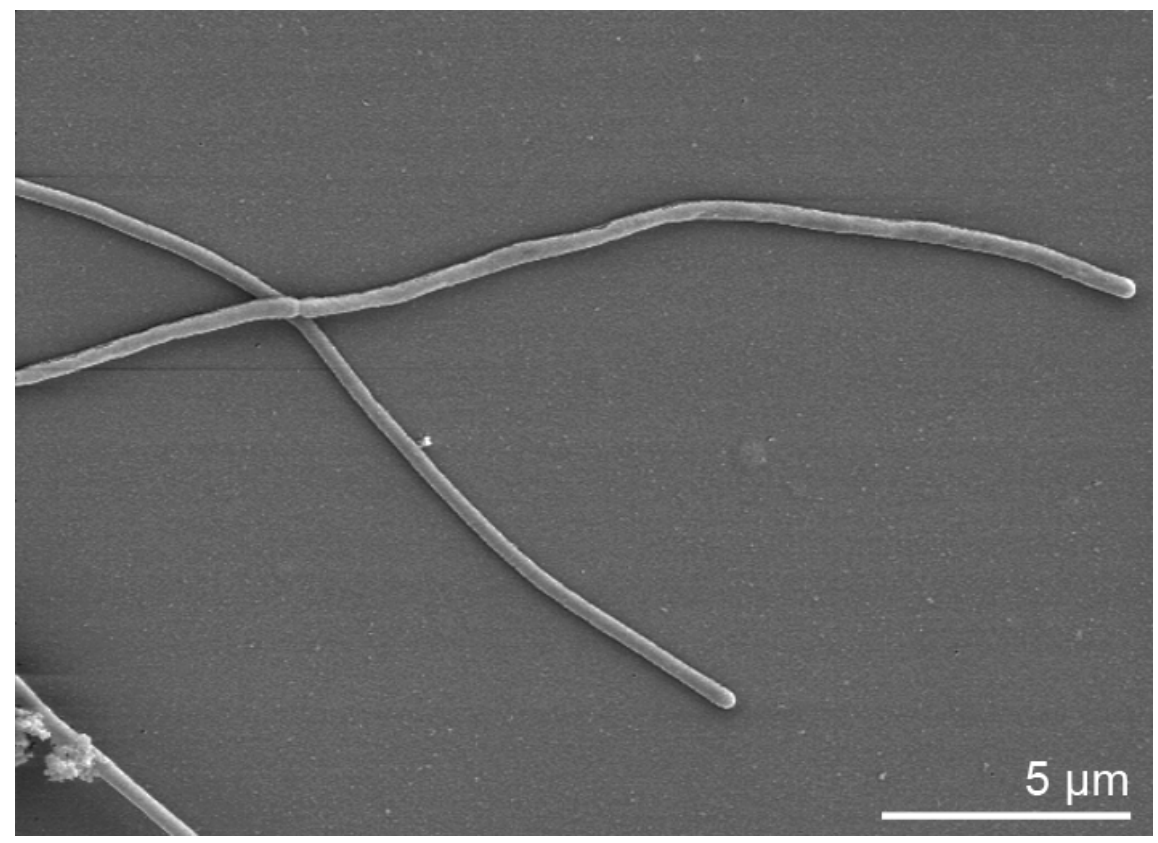

Figure 2. Scanning electron micrograph of C. pinensis UQM 2034

\section{Chemotaxonomy}

The fatty acid profile of strain UQM $2034^{\mathrm{T}}$ revealed $\mathrm{C}_{15: 0 \text { iso }}(30.4 \%)$ and $\mathrm{C}_{16: 1 \omega 5 \mathrm{c}}(33.2 \%)$ as the major fatty acids and $\mathrm{C}_{17: 0 \text { iso-3-0H }}(11.5 \%)$ and $\mathrm{C}_{15: 0}$ iso-3-он (3.1\%) as the major hydroxyl fatty acids. MK-7 is the predominant menaquinone [3]. The polar lipid composition has not been analyzed, but phosphatidylethanolamine is reported for $C$. rupis [7].

\section{Genome sequencing and annotation Genome project history}

This organism was selected for sequencing on the basis of its phylogenetic position, and is part of the Genomic Encyclopedia of Bacteria and Archaea project [17]. The genome project is deposited in the Genome OnLine Database [11] and the complete genome sequence is deposited in GenBank. Sequencing, finishing and annotation were performed by the DOE Joint Genome Institute (JGI). A summary of the project information is shown in Table 2.

\section{Growth conditions and DNA isolation}

C. pinensis UQM 2034', DSM 2588, was grown in DSMZ medium 67 (CY-Medium) [25] at $22^{\circ} \mathrm{C}$. DNA was isolated from $0.5-1 \mathrm{~g}$ of cell paste using Qiagen Genomic 500 DNA Kit (Qiagen, Hilden, Germany), with a modified protocol for cell lysis (st/LALMP), as described in Wu et al. [17].

\section{Genome sequencing and assembly}

The genome was sequenced using a combination of Sanger and 454 sequencing platforms. All general aspects of library construction and sequencing performed at the JGI can be found at the JGI website (http://www.jgi.doe.gov/). 454 Pyrosequencing reads were assembled using the Newbler assembler version 1.1.02.15 (Roche). Large Newbler contigs were broken into 2,046 overlapping fragments of $1,000 \mathrm{bp}$ and 9,925 of them entered into the final assembly as pseudo-reads. The sequences were assigned quality scores based on Newbler consensus q-scores with modifications to account for overlap redundancy and to adjust inflated q-scores. A hybrid 454/Sanger assembly was made using the parallel phrap assembler (High Performance Software, LLC). Possible misassemblies were corrected with Dupfinisher or transposon bombing of bridging clones [18]. Gaps between contigs were closed by editing in Consed, custom primer walk or PCR amplification. A total of 882 Sanger finishing reads were produced to close gaps, to resolve repetitive regions, and to raise the quality of the finished sequence. The error rate of the completed genome sequence is 0.01 in 100,000 nucleotides. Together all sequence types provided $26.3 \times$ coverage of the genome. The final assembly contains 91,161 Sanger and 876,658 pyrosequencing reads. 
Table 2. Genome sequencing project information

\begin{tabular}{lll}
\hline MIGS ID & Property & Term \\
\hline MIGS-31 & Finishing quality & Finished \\
& & $\begin{array}{l}\text { Two Sanger libraries: 8kb pMCL200 and } \\
\text { fosmid pcc1 Fos. }\end{array}$ \\
MIGS-28 & Libraries used & Ane 454 pyrosequence standard library. \\
& & ABI3730, 454 GS FLX \\
MIGS-29 & Sequencing platforms & Newbler, phrap \\
MIGS-31.2 & Sequencing coverage & Prodigal, GenePRIMP \\
MIGS-30 & Assemblers & CP001699 \\
MIGS-32 & Gene calling method & August 08, 2009 \\
& INSDC ID & Gc01083 \\
& Genbank Date of Release & 27951 \\
& GOLD ID & 2501651204 \\
& NCBI project ID & DSM 2588 \\
& Database: IMG-GEBA & Tree of Life, GEBA \\
\hline
\end{tabular}

\section{Genome annotation}

Genes were identified using Prodigal [19] as part of the Oak Ridge National Laboratory genome annotation pipeline, followed by a round of manual curation using the JGI GenePRIMP pipeline (http://geneprimp.jgi-psf.org/) [20]. The predicted CDSs were translated and used to search the National Center for Biotechnology Information (NCBI) nonredundant database, UniProt, TIGRFam, Pfam, PRIAM, KEGG, COG, and InterPro databases. Additional gene prediction analysis and functional annotation was performed within the Integrated Microbial Genomes - Expert Review (http://img.jgi.doe.gov/er) platform [21].

\section{Genome properties}

The genome is $9,127,347$ bp long and comprises one main circular chromosome with a $45.2 \%$ GC content (Table 3 and Figure 3). Of the 7,397 genes predicted, 7,302 were protein coding genes, and 95 RNAs. In addition, 110 pseudogenes were also identified. The majority of the protein-coding genes $(62.4 \%)$ were assigned with a putative function while those remaining were annotated as hypothetical proteins. The distribution of genes into COGs functional categories is presented in Table 4.

Table 3. Genome Statistics

\begin{tabular}{lrr}
\hline Attribute & \multicolumn{1}{c}{ Value } & \% of Total \\
\hline Genome size (bp) & $9,127,347$ & $100.00 \%$ \\
DNA Coding region (bp) & $8,163,680$ & $89.44 \%$ \\
DNA G+C content (bp) & $4,128,036$ & $45.23 \%$ \\
Number of replicons & 1 & \\
Extrachromosomal elements & 0 & \\
Total genes & 7,397 & $100.00 \%$ \\
RNA genes & 95 & $1.28 \%$ \\
rRNA operons & 6 & \\
Protein-coding genes & 7,302 & $98.72 \%$ \\
Pseudo genes & 110 & $1.49 \%$ \\
Genes with function prediction & 4,616 & $62.40 \%$ \\
Genes in paralog clusters & 1,705 & $23.05 \%$ \\
Genes assigned to COGs & 4,451 & $60.17 \%$ \\
Genes assigned Pfam domains & 4,685 & $63.34 \%$ \\
Genes with signal peptides & 2,251 & $30.43 \%$ \\
Genes with transmembrane helices & 1,635 & $22.10 \%$ \\
CRISPR repeats & 0 & \\
\hline
\end{tabular}




\section{Insights from genome sequence}

The predominant characteristic feature of $C$. pinensis is the ability to degrade chitin, a $\beta-1,4-$ glycosidic linked homopolymer of N-acetyl-Dglucosamine and one of the most abundant polysaccharides in nature. It is a component of fungal cell walls and of arthropod exoskeletons. Chitin is degraded by chitinases (EC 3.2.1.14); endochitinases randomly cleave within the chitin molecule and exochitinases hydrolyze diacetylchitobiose from the end of a chitin chain. Diacetylchitobiose is further degraded to $\mathrm{N}$-acetylglucosamine by the action of $\mathrm{N}$-acetylglucosaminidases (EC 3.2.1.52).

These glycosidic bond hydrolyzing enzymes were grouped in glycoside hydrolase (GH) families based on amino acid sequence similarities (http://www.cazy.org) [22]. For the C. pinensis genome 169 glycoside hydrolases belonging to 49 different GH families are predicted; 18 of the predicted glycoside hydrolases belong to $\mathrm{GH}$ family 43 which contains xylosidases, xylanases, arabinanases, arabinofuranosidases and galactosidases.

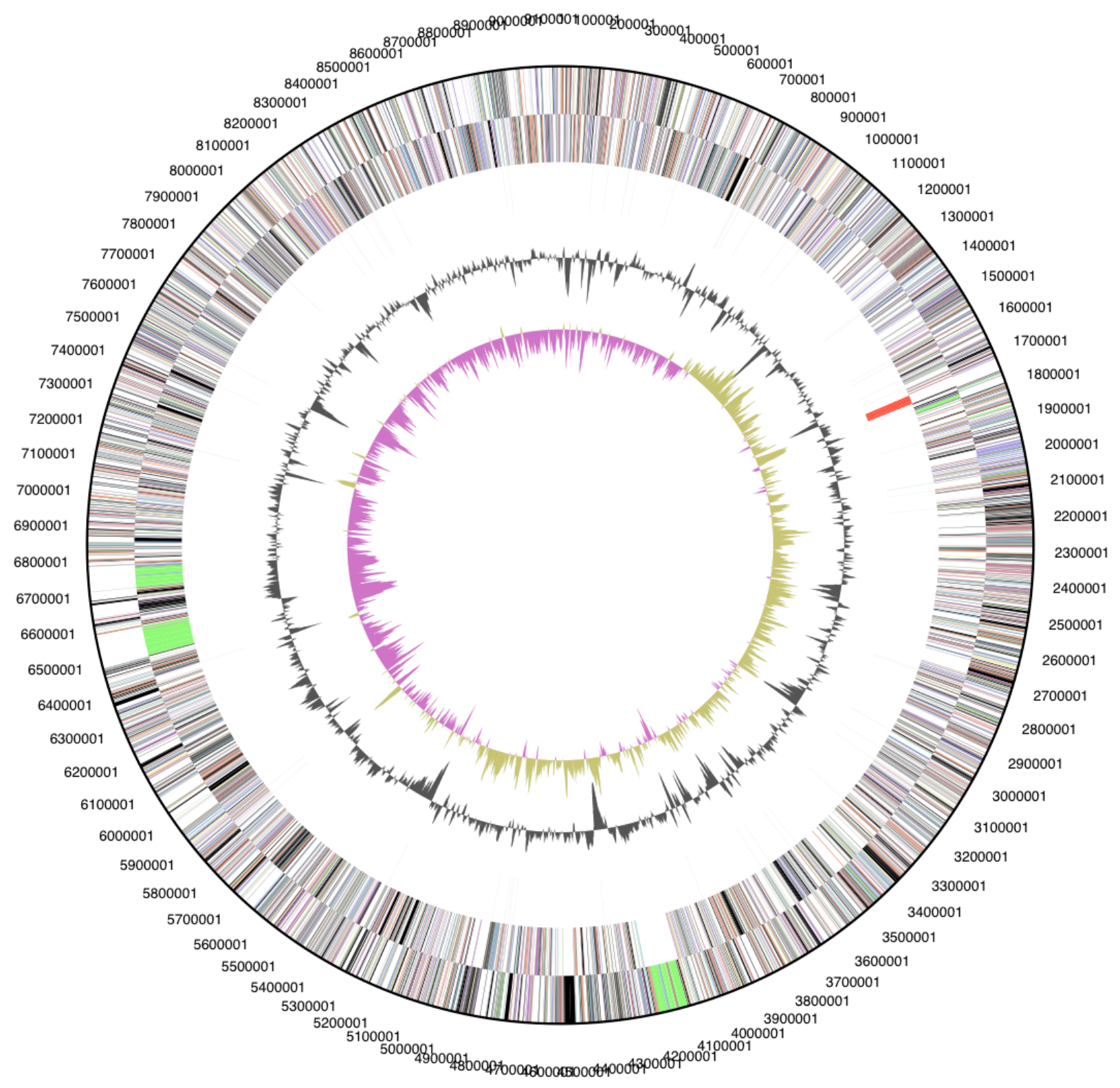

Figure 3. Graphical circular map of the genome. From outside to the center: Genes on forward strand (color by COG categories), Genes on reverse strand (color by COG categories), RNA genes (tRNAs green, rRNAs red, other RNAs black), GC content, GC skew. 
Table 4. Number of genes associated with the general COG functional categories

\begin{tabular}{lrrl}
\hline Code & value & \%age & Description \\
\hline J & 177 & 2.4 & Translation, ribosomal structure and biogenesis \\
A & 0 & 0.0 & RNA processing and modification \\
K & 613 & 8.4 & Transcription \\
L & 192 & 2.6 & Replication, recombination and repair \\
B & 2 & 0.0 & Chromatin structure and dynamics \\
D & 24 & 0.3 & Cell cycle control, mitosis and meiosis \\
Y & 0 & 0.0 & Nuclear structure \\
V & 123 & 1.7 & Defense mechanisms \\
T & 448 & 6.1 & Signal transduction mechanisms \\
M & 337 & 4.6 & Cell wall/membrane biogenesis \\
N & 25 & 0.3 & Cell motility \\
Z & 0 & 0.0 & Cytoskeleton \\
W & 0 & 0.0 & Extracellular structures \\
U & 64 & 0.9 & Intracellular trafficking and secretion \\
O & 170 & 2.3 & Posttranslational modification, protein turnover, chaperones \\
C & 244 & 3.3 & Energy production and conversion \\
G & 330 & 4.5 & Carbohydrate transport and metabolism \\
E & 301 & 4.1 & Amino acid transport and metabolism \\
F & 91 & 1.2 & Nucleotide transport and metabolism \\
H & 210 & 2.9 & Coenzyme transport and metabolism \\
I & 197 & 2.7 & Lipid transport and metabolism \\
P & 328 & 4.5 & Inorganic ion transport and metabolism \\
Q & 146 & 2.0 & Secondary metabolites biosynthesis, transport and catabolism \\
R & 661 & 9.1 & General function prediction only \\
S & 352 & 4.8 & Function unknown \\
- & 2841 & 38.9 & Not in COGs \\
\hline & & & \\
\hline
\end{tabular}

Because of the chitin degrading ability of $C$. pinensis a great number of chitinases was expected to be encoded in the genome. According to the CAZYdatabase, exochitinases and endochitinases belong to $\mathrm{GH}$ families 18, 19 and 48. As estimated, there were several glycoside hydrolases predicted, which may be involved in chitin degradation; five members of GH family 18 (Cpin_2184, Cpin_2186, Cpin_2580, Cpin_3805, Cpin_3919) and three members of GH family 19 (Cpin_5850, Cpin_5553, Cpin_5898). The comparison of the amino acid sequence from these chitinase candidates to the databank BlastP indicated no homologs according to the whole length of the proteins. However, similarities to the known $\mathrm{GH}$ family domains were observed.
The search for $\mathrm{N}$-acetylglucosaminidases (EC 3.2.1.52) in the genome of $C$. pinensis revealed gene Cpin_3944 which encodes a protein with a GH family 20 domain. The predicted GH family 20 domain resembles the well characterized catalytic domain of a N-acetylglucosaminidase from Serratia marcescens [23]. Further $\mathrm{N}$-acetylhexoaminidases of $C$. pinensis are encoded by the genes Cpin_1798, Cpin_4994 and Cpin_1915.

A second way to degrade chitin was described by Davis and Eveleigh in 1984 [24]. First, the chitin molecule is deacetyliated by deacetylases (EC 3.5.1.41), afterwards chitobiose is released from chitosan by the action of chitosanases (EC 3.2.1.132), finally chitobiose is hydrolyzed by glu- 
cosaminidases (EC 3.2.1.52) and glucosamine molecules are released.

One putative chitin deacetylase is encoded in the genome of $C$. pinensis. The deduced amino acid sequence of Cpin_6813 shows a GH family 19 domain and a C-terminal deacetylase domain. Chitosanases that are responsible for the hydrolysis of chitosan are mainly found in GH family 46 but also occur in GH families 5 and 18. In C. pinensis, no GH

\section{Acknowledgements}

We would like to gratefully acknowledge the help of Birgit Merkhoffer for growing C. pinensis cultures and Susanne Schneider for DNA extraction and quality analysis (both at DSMZ). This work was performed under the auspices of the US Department of Energy's Office of Science, Biological and Environmental Research Program, and by the University of California,

\section{References}

1. Sangkhobol V, Skerman VBD. Chitinophaga, a new genus of chitinolytic myxobacteria. Int / Syst Bacteriol 1981; 31:285-293.

2. Sly LI, Taghavi M, Fegan M. Phylogenetic position of Chitinophaga pinensis in the FlexibacterBacteroides-Cytophaga phylum. Int I Syst Bacteriol 1999; 49:479-481. PubMed

3. Kämpfer P, Young CC, Sridhar KR, Arun AB, Lai WA, Shen FT, Rekha PD. Transfer of [Flexibacter] sancti, [Flexibacter] filiformis, [Flexibacter] japonensis and [Cytophaga] arvensicola to the genus Chitinophaga and description of Chitinophaga skermanii sp. nov. Int I Syst Evol Microbiol 2006; 56:2223-2228. PubMed doi:10.1099/ijs.0.64359-0

4. Lee HG, An DS, Im WT, Liu QM, Na JR, Cho DH, Jin CW, Lee ST, Yang DC. Chitinophaga ginsengisegetis sp. nov. and Chitinophaga ginsengisoli sp. nov., isolated from soil of a ginseng field in South Korea. Int J Syst Evol Microbiol 2007; 57:13961401. PubMed doi:10.1099/ijs.0.64688-0

5. Kim MK, Jung HY. Chitinophaga terrae sp. nov., isolated from soil. Int J Syst Evol Microbiol 2007; 57:1721-1724. PubMed doi:10.1099/ijs.0.64964-0

6. Weon HY, Yoo SH, Kim YJ, Son JA, Kim BY, Kwon SW, Koo BS. Chitinophaga niabensis sp. nov. and Chitinophaga niastensis sp. nov., isolated from soil. Int / Syst Evol Microbiol 2009; 59:1267-1271. PubMed PubMed doi:10.1099/ijs.0.004804-0 family 46 members were observed, but the presence of nine GHs belonging to family 5 and five members of GH family 18 are predicted. One of these glycoside hydrolases might have a chitosanase function. It remains unclear which pathway C. pinensis uses for the degradation of chitin and whether the predicted functions of the proteins match the real functions.

Lawrence Berkeley National Laboratory under contract No. DE-AC02-05CH11231, Lawrence Livermore National Laboratory under Contract No. DE-AC5207NA27344, and Los Alamos National Laboratory under contract No. DE-AC02-06NA25396, as well as German Research Foundation (DFG) INST 599/1-1.

7. Lee DW, Lee JE, Lee SD. Chitinophaga rupis sp. nov., isolated from soil. Int I Syst Evol Microbiol 2009; 59:2830-2833. PubMed doi:10.1099/ijs.0.011163-0

8. Lee C, Grasso C, Sharlow MF. Multiple sequence alignment using partial order graphs. Bioinformatics 2002; 18:452-464. PubMed doi:10.1093/bioinformatics/18.3.452

9. Castresana J. Selection of conserved blocks from multiple alignments for their use in phylogenetic analysis. Mol Biol Evol 2000; 17:540-552. PubMed

10. Stamatakis A, Hoover P, Rougemont J. A Rapid Bootstrap Algorithm for the RAxML Web Servers. Syst Biol 2008; 57:758-771. PubMed doi:10.1080/10635150802429642

11. Liolios K, Chen IM, Mavromatis K, Tavernarakis $\mathrm{N}$, Hugenholtz P, Markowitz VM, Kyrpides NC. The Genomes On Line Database (GOLD) in 2009: status of genomic and metagenomic projects and their associated metadata. Nucleic Acids Res 2010; 38:D346-D354. PubMed doi:10.1093/nar/gkp848

12. Field D, Garrity G, Gray T, Morrison N, Selengut J, Sterk P, Tatusova T, Thomson N, Allen MJ, Angiuoli SV, et al. The minimum information about a genome sequence (MIGS) specification. Nat Biotechnol 2008; 26:541-547. PubMed doi:10.1038/nbt1360

13. Woese CR, Kandler O, Wheelis ML. Towards a natural system of organisms. Proposal for the do- 
mains Archaea and Bacteria. Proc Natl Acad Sci

USA 1990; 87:4576-4579. PubMed

doi:10.1073/pnas.87.12.4576

14. Garrity GM, Holt JG. Taxonomic Outline of the Archaea and Bacteria. In: Garrity GM, Boone DR, Castenholz RW (eds), Bergey's Manual of Systematic Bacteriology, Second Edition, Volume 1, Springer, New York, 2001, p. 155-166

15. Biological Agents. Technical rules for biological agents www.baua.de TRBA 466.

16. Ashburner M, Ball CA, Blake JA, Botstein D, Butler H, Cherry JM, Davis AP, Dolinski K, Dwight SS, Eppig JT, et al. Gene ontology: tool for the unification of biology. The Gene Ontology Consortium. Nat Genet 2000; 25:25-29. PubMed doi:10.1038/75556

17. Wu D, Hugenholtz P, Mavrommatis K, Pukall R, Dalin E, Ivanova N, Kunin V, Goodwin L, Wu M, Tindall BJ, et al. A phylogeny-driven genomic encyclopedia of Bacteria and Archaea. Nature 2009; 462:1056-1060. PubMed doi:10.1038/nature08656

18. Sims D, Brettin T, Detter JC, Han C, Lapidus A, Copeland A, Glavina Del Rio T, Nolan M, Chen F, Lucas S, et al. Complete genome of Kytococcus sedentarius type strain $\left(541^{\top}\right)$. Stand Genomic Sci 2009; 1:12-20. doi:10.4056/sigs.761

19. Hyatt D, Chen G-L, LoCascio PF, Land ML, Larimer FW, Hauser LJ. Prodigal: prokaryotic gene recognition and translation initiation site identification. BMC Genomics (In press)
20. Pati A, Ivanova N, Mikhailova, N, Ovchinikova G, Hooper SD, Lykidis A, Kyrpides NC. GenePRIMP: A Gene Prediction Improvement Pipeline for microbial genomes. Nature Methods (In press).

21. Markowitz VM, Mavromatis K, Ivanova NN, Chen IMA, Chu K, Kyrpides NC. IMG ER: a system for microbial genome annotation expert review and curation. Bioinformatics 2009; 25:2271-2278. PubMed doi:10.1093/bioinformatics/btp393

22. Cantarel BL, Coutinho PM, Rancurel C, Bernard T, Lombard V, Henrissat B. The CarbohydrateActive EnZymes database (CAZy): an expert resource for Glycogenomics. Nucleic Acids Res 2009; 37:D233-D238. PubMed doi:10.1093/nar/gkn663

23. Prag G, Papanikolau Y, Tavlas G, Vorgias CE, Petratos K, Oppenheim AB. Structures of chitobiase mutants complexed with the substrate di-Nacetyl-D-glucosamine: the catalytic role of the conserved acidic pair, aspartate 539 and glutamate 540. J Mol Biol 2000; 300:611-617. PubMed doi:10.1006/jmbi.2000.3906

24. Davis B, Eveleigh DE. Chitosanases: occurrence, production and immobilization. In: Zikalis JP (ed.), Chitin, Chitosan and Related Enzymes. Academic Press 1984;161-179.

25. List of growth media used at DSMZ: http://www.dsmz.de/microorganisms/media_list.p $\underline{\mathrm{hp}}$ 